Review

\title{
Viroid Replication: Rolling-Circles, Enzymes and Ribozymes
}

\section{Ricardo Flores*, María-Eugenia Gas, Diego Molina-Serrano, María-Ángeles Nohales, Alberto Carbonell, Selma Gago, Marcos De la Peña and José-Antonio Daròs}

Instituto de Biología Molecular y Celular de Plantas (UPV-CSIC), Avenida de los Naranjos s/n, 46022 Valencia, Spain; E-Mails: mgas@ibmcp.upv.es (M.-E.G.); dmolina@ibmcp.upv.es (D.M.-S.); manozaf@ibmcp.upv.es (M.-A.N.); alcarol@ibmcp.upv.es (A.C.); selmag@ibmcp.upv.es (S.G.); rivero@ibmcp.upv.es (M.D-P.)

*Author to whom correspondence should be addressed; E-Mail: rflores@ibmcp.upv.es; Tel.: +34-963-877-861; Fax: +34-963-877-859.

Received: 6 July 2009; in revised form: 9 September 2009 / Accepted: 9 September 2009 / Published: 14 September 2009

\begin{abstract}
Viroids, due to their small size and lack of protein-coding capacity, must rely essentially on their hosts for replication. Intriguingly, viroids have evolved the ability to replicate in two cellular organella, the nucleus (family Pospiviroidae) and the chloroplast (family Avsunviroidae). Viroid replication proceeds through an RNA-based rolling-circle mechanism with three steps that, with some variations, operate in both polarity strands: i) synthesis of longer-than-unit strands catalyzed by either the nuclear RNA polymerase II or a nuclear-encoded chloroplastic RNA polymerase, in both instances redirected to transcribe RNA templates, ii) cleavage to unit-length, which in the family Avsunviroidae is mediated by hammerhead ribozymes embedded in both polarity strands, while in the family Pospiviroidae the oligomeric RNAs provide the proper conformation but not the catalytic activity, and iii) circularization. The host RNA polymerases, most likely assisted by additional host proteins, start transcription from specific sites, thus implying the existence of viroid promoters. Cleavage and ligation in the family Pospiviroidae is probably catalyzed by an RNase III-like enzyme and an RNA ligase able to circularize the resulting 5' and 3' termini. Whether a chloroplastic RNA ligase mediates circularization in the family Avsunviroidae, or this reaction is autocatalytic, remains an open issue.
\end{abstract}

Keywords: viroids; catalytic RNAs; hammerhead ribozymes 


\section{Introduction}

Perhaps the most striking aspect of viroids from a functional perspective is that, in contrast to viruses, they lack protein-coding ability [1,2]. This feature directly linked to their structural peculiarities - viroids are RNAs of 246-301 nt that would limit the size of their potential translation products and, additionally, with a circular structure that would impede translation by a scanning mechanism starting from the 5' terminus - has deep consequences in the context of replication. While RNA viruses encode subunits of the enzymatic complex (RNA replicase) that catalyzes initiation and elongation of viral RNA strands, viroids must rely for this replication step on pre-existing host RNA polymerases [2-7]. In principle, the best candidates would be RNA-dependent RNA polymerases, whose existence in plants has been known for a long time [8,9]. However, viroids do not use these enzymes for their replication, but DNA-dependent RNA polymerases redirected to accept RNA templates (see below). Why and how viroids have evolved this capacity is one of the most intriguing issues awaiting an answer. Additionally, some viroids have evolved a second outstanding capacity: they are catalytic RNAs "encoding” in their strands hammerhead ribozymes that play a crucial role in replication. Therefore, studies on how viroids replicate have revealed aspects of RNA metabolism that were totally unanticipated, some of which might also play a role in their hosts. Illustrating this point, naturally-encoded hammerhead ribozymes have been discovered in the genome of Arabidopsis thaliana [10] and mouse [11]; the latter, embedded in a messenger RNA, is able to reduce protein expression in vivo.

\section{An overview of viroid singularity and diversity}

Replication of viroids is very much dependent of their intrinsic properties and of the different subcellular organella in which this process occurs. Sequence and structural analyses of the approximately 30 viroids characterized so far have revealed that they can be clustered into two families: i) Pospiviroidae, type species Potato spindle tuber viroid (PSTVd), the members of which exhibit a central conserved region (CCR) and most adopt a rod-like secondary struture, and ii) Avsunviroidae, type species Avocado sunblotch viroid (ASBVd), whose members lack a CCR but have the ability to form hammerhead ribozymes in both polarity strands, and fold into quasi-rod-like or clearly branched secondary structures. Allocation of viroid species into genera is based on additional criteria. The two families also differ regarding their functional properties: the available evidence indicates that while members of the family Pospiviroidae have a nuclear replication (and accumulation), those of the family Avsunviroidae replicate (and accumulate) in the chloroplast $[4,12]$. These very different subcellular sites have deep implications on the enzymology underlying viroid replication. Viroids replicate through an RNA-based rolling-circle mechanism with three steps that, with some variations, operate in the strands of both polarities: i) synthesis of longer-than-unit strands catalyzed by a host nuclear or chloroplastic RNA polymerase that reiteratively transcribes the initial circular template, ii) processing to unit-length, which remarkably is mediated by hammerhead ribozymes in the family Avsunviroidae, and iii) circularization resulting from the action of an RNA ligase or occuring autocatalytically (Figure 1).

Viroids share structural and functional similarities with the so-called viroid-like satellite RNAs (small, circular, non-protein-coding genomes that replicate through a rolling-circle mechanism 
involving ribozymes) but, in contrast to viroids, viroid-like satellite RNAs depend for their replication on a helper virus [13]. The RNA of Human hepatitis delta virus also bears some resemblance with viroids (circularity and replication through a rolling-circle mechanism mediated by ribozymes), although it has a larger size and encodes a protein in its antigenomic polarity [14]. How viroids, with such a tiny genome, are able to promote their own replication? Here we will review hypotheses and results on this issue, putting special emphasis on those most recent that have challenged some longheld tenets.

\section{Family Pospiviroidae: an asymmetric rolling-circle mechanism with circular and linear templates catalyzed by host enzymes}

The basis for this model were founded by early experiments performed with PSTVd and, to a lesser extent, with other members of the same genus (Pospiviroid) $[4,15,16]$. Replication starts when the incoming monomeric circular $(m c)$ RNA — to this most abundant species in vivo is assigned the $(+)$ polarity by convention - is reiteratively transcribed into oligomeric (-) RNAs that in turn serve as templates for synthesis of oligomeric $(+)$ RNAs. These latter transcripts are then cleaved into the monomeric linear $(\mathrm{ml})$ form and ligated to the mature $m c(+)$ RNA, the final product of the cycle (Figure 1). This mechanism, which is termed asymmetric because the rolling-circle only operates for synthesis of the oligomeric (-) RNAs, is catalyzed by host enzymes that recognize specific RNA motifs. Assuming that the loops and bulges (flanked by double-stranded helices) predicted in the PSTVd secondary structure are functional motifs that regulate replication in single cells or systemic trafficking in planta, a recent genome-wide mutational analysis has identified multiple loops/bulges essential or important for each of these biological processes [17]. The resulting genomic map indicates that motifs critical for replication are concentrated in the CCR and in the left terminal domain. This view is supported by additional independent data (see below).

\subsection{Initiation and elongation: involvement of RNA polymerase II}

Two lines of evidence support that the enzyme catalyzing elongation of PSTVd and closely-related viroids is RNA polymerase II: i) nanomolar concentrations of the fungal toxin $\alpha$-amanitin that typically inhibit this enzyme also block in vivo and in vitro transcription of $(+)$ and (-) viroid strands, and ii) viroid $(+)$ and $(-)$ strands have been retrieved with a monoclonal antibody against the carboxyterminal domain of the largest subunit of RNA polymerase II [4].

Regarding the transcription initiation sites, this question has been recently tackled by priming a nuclear extract from a non-infected cell culture of the host plant Solanum tuberosum with the PSTVd $m c(+)$ RNA. Following purification by affinity chromatography, analysis by primer extension of the (-) strands synthesized de novo revealed a single start site located in the hairpin loop of the left terminal domain of the rod-like secondary structure proposed for PSTVd $m c(+)$ RNA [18]. Further analyses in planta of site-directed mutants are consistent with this hairpin loop being involved in infectivity and perhaps replication [18], as also is a genome-wide mutational analysis [17]. However, additional studies are needed to confirm this site as well as to identify the transcription initiation site of (+) strands, which remains unknown. The possibility exists that, like other RNA polymerase II 
transcripts, the 5' termini of (+) and (-) RNAs of nuclear viroids could be capped in vivo, a signature that would mark unambiguously their initiation sites.

Figure 1. Rolling-circle replication mechanism with the two alternative pathways followed by members of the family Pospivirodae (asymmetric) and Avsunviroidae (symmetric) $[15,16,46]$. Red and blue lines refer to $(+)$ and (-) strands, respectively. Arrowheads mark the cleavage sites of a host RNase III or a hammerhead ribozymes (Rz), and the resulting terminal groups are indicated. Elongation of RNA strands is catalyzed by the nuclear RNA polymerase II (RNA pol II) or the nuclear-encoded chloroplastic RNA polymerase (NEP).

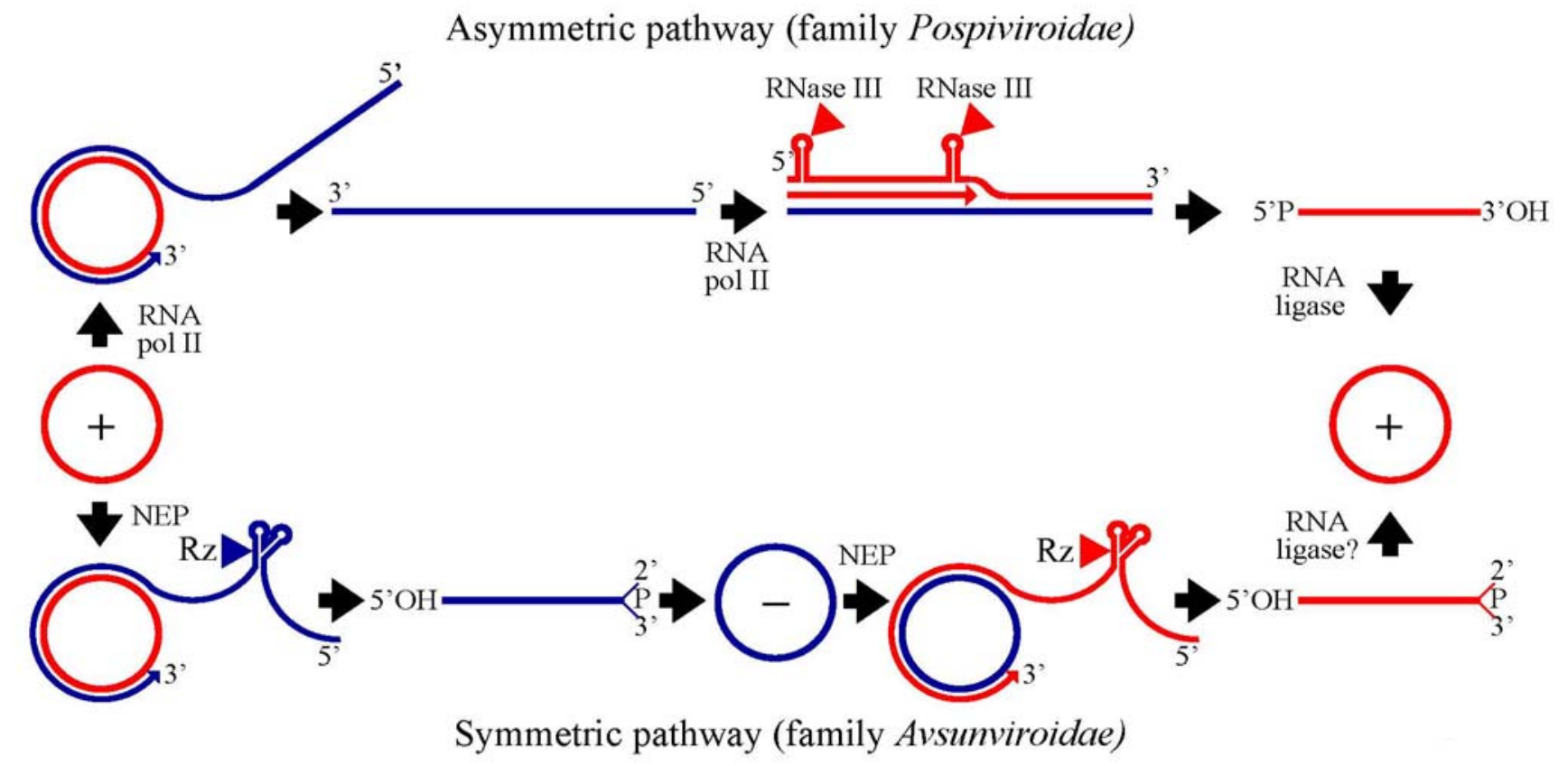

\subsection{Cleavage of oligomeric (+) strands is most likely mediated by a member of the RNase III family}

Early studies implicated the CCR in processing of the oligomeric $(+)$ strands of the family Pospiviroidae either through hairpin I, a metastable motif that can be formed by the upper CCR strand and flanking nucleotides during thermal denaturation [19], or through a thermodynamically stable double-stranded structure that can be alternatively assumed by the same sequences in oligomeric RNAs. Data supporting this view include infectivity bioassays with different PSTVd DNA and RNA constructs [20], with longer-than-unit clones of Hop stunt viroid (HSVd) [21], and with constructs of Citrus exocortis viroid (CEVd) containing sequence repetitions and point mutations in the upper CCR strand [22]. A critical reassessment of these data led to a model involving the double-stranded structure in cleavage, although the model did not predict the mechanism of cleavage-ligation or specify the exact processing site [23]. On the other hand, in vitro and thermodynamic analyses of the products obtained by incubating a potato nuclear extract with a full-length PSTVd RNA containing a 17-nt repeat of the upper CCR strand led to propose that cleavage of $(+)$ strands is driven by a multibranched structure with a hairpin - different from hairpin I - capped by a GAAA tetraloop conserved in members of the genus Pospiviroid. This multibranched structure subsequently switches to an extended conformation with a loop E (see below) that promotes ligation [24]. Yet, the processing complex 
formed in vitro may not mimic the corresponding complex in vivo. Moreover, other members of the family Pospiviroidae cannot form the hairpin capped by a GAAA tetraloop [4], and alternative processing sites in the lower CCR strand, or outside this region, have been observed for several members of this family [25].

This question has been recently examined in vivo [26], using a system based in transgenic $A$. thaliana expressing dimeric $(+)$ transcripts, $d t(+)$ RNAs, of CEVd, HSVd, and Apple scar skin viroid (ASSVd) [27] of the genera Pospiviroid, Hostuviroid, and Apscaviroid, respectively, within the family Pospiviroidae [4]. This system circumvents most of these limitations. It is an in vivo system in which processing is correct: transgenically expressed dimeric transcripts of typical members of the family Pospiviroidae are cleaved to the $\mathrm{ml}$ forms and then ligated to the infectious $m c$ RNAs, whereas the complementary $d t$ (-) RNAs are not [26,27], thus reproducing the situation observed in typical hosts. However, in contrast to typical hosts in which turnover of the longer-than-unit $(+)$ replicative intermediates is difficult to follow because of their low accumulation and diverse size, the $A$. thalianabased system provides a constant supply of a size-specific replicative-like intermediate that can be easily quantified as well as its processing products. Moreover, the $m l$ and $m c(+)$ RNAs can be assumed to come essentially from processing of the transgenically expressed $d t(+)$ RNA. Therefore, the effects of specific mutations in the primary transcript on cleavage and ligation can be evaluated regardless of whether the resulting products are infectious or not - and it is even possible to identify mutations affecting only ligation (see below).

Results with the A. thaliana-based system have mapped the cleavage site of CEVd (+) strands at the upper CCR strand [26], in a position equivalent to that inferred for PSTVd with an in vitro system [24]. However, the RNA motif directing cleavage in vivo does not seem to be the GAAA-capped hairpin proposed previously [24], but the hairpin I/double-stranded structure [26]. The first argument supporting this view is that whereas the cleavage sites of HSVd and ASSVd (+) strands also map at equivalent positions in a similar hairpin I/double-stranded structure, these viroids cannot form the GAAA-capped hairpin. In contrast, examination of the hairpin I/double-stranded structure reveals some appealing features. Hairpin I is composed by a tetraloop, a 3-bp stem, an internal symmetric loop of 1-3 nt in each strand that presumably interact by non-Watson-Crick base pairs [28], and a 9-10-bp stem that can be interrupted by a 1-nt symmetric or asymmetric internal loop [22,25] (Figure 2). Remarkably, these structural features are conserved in the type species of the five genera composing the family Pospiviroidae and additionally: i) the capping tetraloop is palindromic itself, and ii) the two central positions of the tetraloop and the central base pair of the 3-bp stem are phylogenetically conserved (Figure 2) [25]. As a consequence, a long double-stranded structure with a GC-rich central region of $10 \mathrm{bp}$ containing the cleavage sites can be alternatively assumed by the same sequences in a di- or oligomeric RNA (Figure 3). The second argument supporting the hairpin I/double-stranded structure as the RNA motif directing cleavage derives from the effects on this reaction of different CEVd mutants expressed transgenically in A. thaliana affecting differentially this motif versus the GAAA-capped hairpin [26]. 
Figure 2. Hairpin I structures of the five type species of the family Pospiviroidae. This structural element is formed by the upper CCR strand and flanking nucleotides of the type members of the five genera composing the family Pospiviroidae: PSTVd, HSVd, CCCVd (Coconut cadang-cadang viroid), ASSVd, and CbVd1 (Coleus blumei viroid 1) [25,26]. Red fonts indicate conserved nucleotides in structurally similar positions. Continuous and broken lines represent Watson-Crick and non-canonical base pairs, respectively [28]. Notice that the variability preserves the overall structure of hairpin I, including the terminal palindromic tetraloop, the adjacent 3-bp stem, and the long stem. Left inset, hairpin I of the CEVd variant used to transform A. thaliana [26]; notice two covariations with respect to PSTVd at the basis of the long stem. Reprinted with permission from [26].

\begin{tabular}{|c|c|c|c|c|c|}
\hline CEVd & PSTVd & HSVd & $\mathrm{CCCVd}$ & ASSVd & $\mathrm{CbVd1}$ \\
\hline C $\mathbf{G}^{96}$ & C G95 & C $\mathrm{G} 82$ & C $G^{62}$ & C $G 90$ & C G73 \\
\hline C $\quad G$ & C $\quad G$ & C $\quad G$ & C $\quad G$ & $\mathbf{U} \quad \mathbf{A}$ & G $\quad$ C \\
\hline$C-G$ & $C-G$ & $C-G$ & $C-G$ & G-C & $\mathrm{A}-\mathrm{U}$ \\
\hline$C-G$ & $C-G$ & $\mathrm{C}-\mathrm{G}$ & $\mathrm{C}-\mathrm{G}$ & $\mathrm{C}-\mathrm{G}$ & $\mathrm{C}-\mathrm{G}$ \\
\hline $\mathrm{U}-\mathrm{A} 100$ & $\mathrm{U}-\mathrm{A}$ & $G-C$ & $\mathrm{U}-\mathrm{A}$ & $\mathrm{U}-\mathrm{A}$ & $G-C$ \\
\hline $90 \mathrm{~A} \cdot \mathrm{A}$ & $\mathrm{A} \cdot \mathrm{A}$ & $A \cdot A$ & $\mathbf{A} \cdot \mathrm{A}_{\mathrm{A}}$ & $\mathbf{G} \cdots \mathbf{A}$ & $\mathbf{G} \cdots \mathbf{A}$ \\
\hline $\mathbf{G} \cdot \boldsymbol{A}_{\mathbf{A}}$ & $\mathbf{G} \cdot \boldsymbol{A}_{\mathbf{A}}$ & $\mathbf{G} \cdot \boldsymbol{A}_{\mathbf{A}}$ & $\mathbf{G} \cdot \boldsymbol{A}_{\mathbf{A}}$ & $C-G$ & $\mathrm{U} \cdots \mathbf{A}$ \\
\hline$G-C$ & $G-C$ & $G-C$ & G-C & $\mathrm{U}-\mathrm{G}$ & C. . C 80 \\
\hline$G-C$ & $G-C$ & $\mathrm{~A}-\mathrm{U} 90$ & G-C70 & $G-C$ & $C-G$ \\
\hline $\mathrm{A}-\mathrm{U}$ & $A-U$ & $A-U^{C}$ & $A-U$ & $80 \mathrm{U} \cdot \mathrm{C}$ & $C-G$ \\
\hline$C-G$ & $C-G$ & $\mathrm{~A}-\mathrm{U}$ & G-C & C-G 100 & $\mathrm{U}-\mathrm{A}$ \\
\hline U-G & U-G & $70 \mathrm{G}-\mathrm{C}$ & $50 \mathrm{U}-\mathrm{A}$ & $C-G$ & $\mathrm{U}-\mathrm{A}$ \\
\hline $\mathrm{U}-\mathrm{A}$ & $\mathrm{U}-\mathrm{A}$ & $A-U$ & $U-A$ & $\mathrm{~A}-\mathrm{U}$ & $60 \mathrm{G}-\mathrm{U}$ \\
\hline$C-G$ & $C-G$ & G-C & C-G & C-G & G-C \\
\hline C-G110 & $80 \mathrm{G}-\mathrm{C}$ & $\mathrm{U}-\mathrm{A}$ & G-C & $\mathrm{U}-\mathbf{A}$ & G-C \\
\hline & G 110 & & & & $\begin{array}{l}U-A \\
C-G\end{array}$ \\
\hline
\end{tabular}

Altogether, the results obtained with the A. thaliana-based system and with some experimental hosts [26] indicate that the substrate for cleavage in vivo of all members of the family Pospiviroidae is the double-stranded structure proposed previously [23], with hairpin I playing a role in promoting the adoption of this structure (see below). Moreover, the cleavage sites in the double-stranded structure leave two 3'-protruding nucleotides in each strand (Figure 3), the characteristic signature of RNase III enzymes $[29,30]$. The participation of an enzyme of this class, of which there are at least seven in $A$. thaliana [31], is consistent with the nuclear location of some of them, which additionally have preference for substrates with a strong secondary structure resembling that of viroids. Going one step further, if an RNase III indeed catalyzes cleavage of the oligomeric (+) RNAs of the family Pospiviroidae, the resulting products should have 5'-phosphomonoester and free 3'-hydroxyl termini. Characterization of the $m l(+)$ RNAs from $A$. thaliana transgenically expressing $d t$ CEVd $(+)$ RNAs has shown that this is actually the case [32]. The adoption in vivo of the double-stranded structure with a GC-rich central region containing the cleavage sites could be promoted by hairpin I because prior work with PSTVd has mapped a dimerization domain at this hairpin [28]. This situation resembles that observed previously in retroviruses in which dimerization, a critical step of their infectious cycle, is mediated by a hairpin with a palindromic loop that can dimerize via a kissing loop interaction [33]. 
During transcription of oligomeric (+) RNAs of the family Pospiviroidae, a kissing loop interaction between the palindromic tetraloops of two consecutive hairpin I motifs might similarly start intramolecular dimerization, with their stems then forming a longer interstrand duplex [32] (Figure 3).

Figure 3. Model for processing in vivo of the oligomeric $(+)$ replicative intermediates of the family Pospiviroidae that involves a kissing loop interaction between the palindromic tetraloops of two consecutive hairpin I motifs (A), with their stems forming subsequently a longer interstrand duplex (B). This double-stranded structure is the substrate for cleavage at specific positions in both strands (C). Following a second conformational switch the resulting unit-length strands adopt the extended rod-like structure with loop E (in outlined fonts) and the adjacent bulged-U helix (D), which is the substrate for ligation (E). R and Y refer to purines and pyrimidines, respectively, the S-shaped line denotes the UV-induced cross-link, and white arrowheads mark the cleavage sites in the double-stranded structure and the ligation site in the extended conformation. Reprinted with permission from [26].

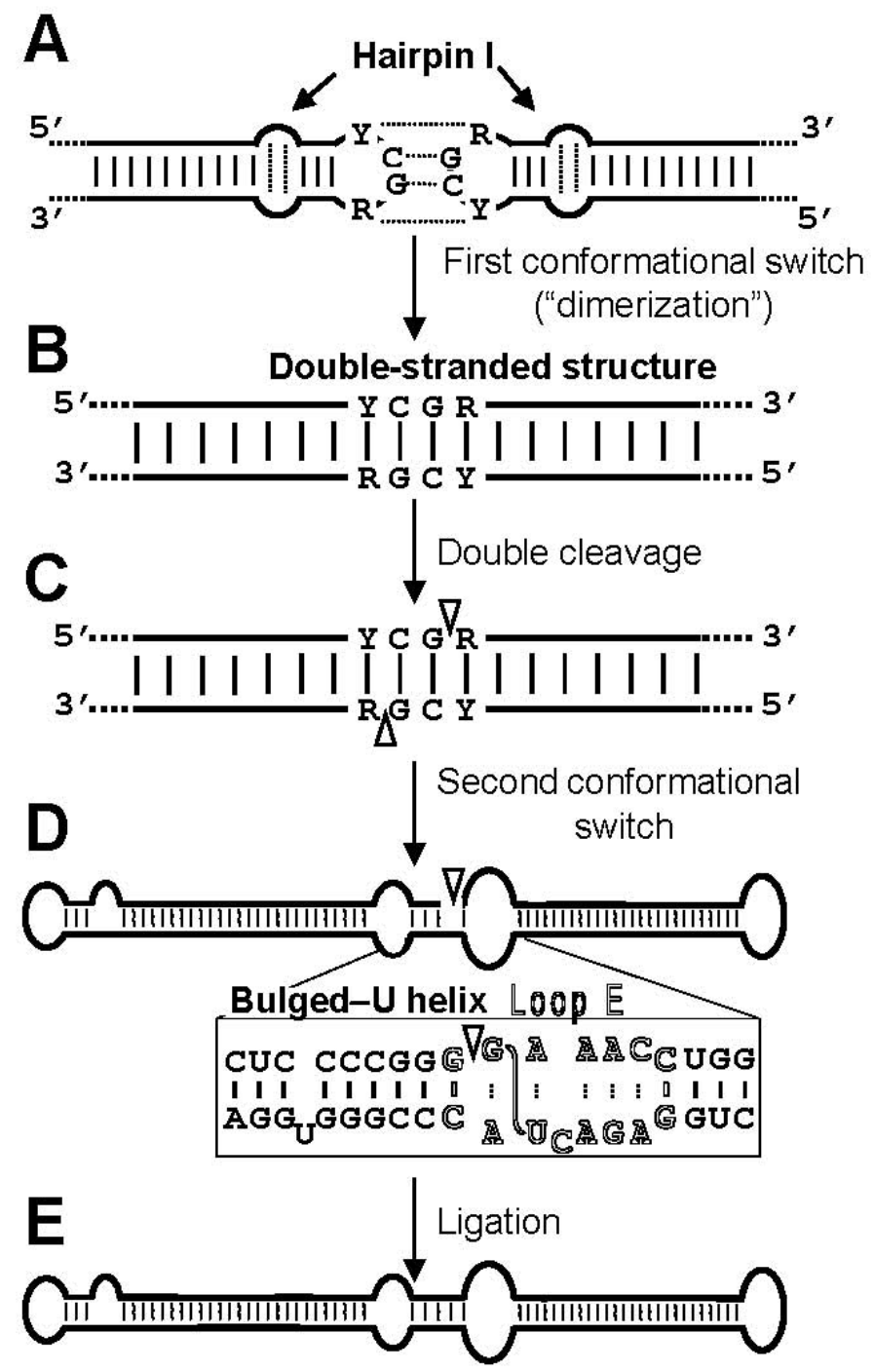




\subsection{Ligation of monomeric linear (+) strands: presumable participation of a novel RNA ligase}

Initial in vitro assays showed that incubation with wheat germ RNA ligase converts the monomeric linear (+) PSTVd RNA isolated from infected tissue into its bona fide circular counterpart, suggesting that the reaction might be mediated by an enzyme of this class [34], which demands 5'-hydroxyl and 2',3'-cyclic phosphodiester termini [35]. Although subsequent experiments with the fungal RNase T1 revealed that it can process in vitro linear oligomeric (+) PSTVd RNAs into infectious monomeric circular RNA [36], it is unlikely that an RNAse could catalyze in vivo both cleavage and ligation. Moving into a more physiological context, incubation with a potato nuclear extract of a monomeric $(+)$ PSTVd RNA with a short repeat of the CCR upper strand produced the infectious monomeric circular form, leading to the proposal that the enzymatic cleavage and ligation of the PSTVd $(+)$ strand is driven by a switch from a branched structure containing a GAAA-capped hairpin (see above) to an extended conformation with an E loop [24]. Loop E, a UV-sensitive motif of RNA tertiary structure that is conserved in PSTVd and members of its genus [4] and exists in vitro [37] and in vivo [38,39], has also been involved in host specificity [40], pathogenesis [41], and transcription [42]. A structural model based on isostericity matrix and mutagenic analyses has been derived recently for PSTVd loop E [42]; this model can be extended to other closely-related viroids like CEVd.

With the aim of circumventing the intrinsic limitations of in vitro approaches, the most important of which is to what extent results from these approaches can be extrapolated to the in vivo situation, the A. thaliana-based system [27] described in the previous section for studying in vivo cleavage of the oligomeric $(+)$ strands has also been used to examine the requisites for proper ligation of the resulting $m l(+)$ RNAs. Data obtained with this system indicate that the substrate for this reaction in the genus Pospiviroid is the extended conformation containing loop E [26] (Figure 3). Therefore, whereas cleavage is only dependent on the upper CCR strand and flanking nucleotides, ligation is dependent on nucleotides of both CCR strands that encompass those of loop E and others adjacent. Because within the family Pospiviroidae loop E is only formed in the genera Pospiviroid and Cocadviroid, other genera of this family must have alternative motifs playing a similar role in ligation. Potential candidates are the extended conformation of the CCR with a bulged-U helix conserved in all members of the genera Pospiviroid, Hostuviroid and Cocadviroid (Figure 3D), and similar structures in the other genera of this family. More recent data, obtained by rapid amplification of 5' and 3' cDNA ends and in vitro ligation assays with the T4 RNA ligase 1 and $A$. thaliana tRNA ligase, have shown that the $m l$ $\mathrm{CEVd}(+)$ RNA resulting from cleavage of a dimeric transcript transgenically expressed in $A$. thaliana indeed contains the 5'-phosphomonoester and 3'-hydroxyl termini expected from cleavage mediated by an RNase III [32]. Therefore, unless the termini are later modified, these results suggest the existence of a second plant RNA ligase that would mediate joining of the same 5'-P and 3'-OH termini as those required by the T4 RNA ligases 1 and $2[43,44]$.

\section{Family Avsunviroidae: a symmetric rolling-circle mechanism with circular RNA templates catalyzed by host enzymes and viroid ribozymes}

This model is founded in results obtained with ASBVd $[4,12,45,46]$, additionally supported with data generated with the other members of the family: Peach latent mosaic viroid (PLMVd) [47], Chrysanthemum chlorotic mottle viroid (CChMVd) [48] and Eggpant latent viroid (ELVd) [49]. In 
this family, the oligomeric (-) RNAs produced in the first rolling-circle are cleaved and ligated into the $m c(-)$ form, then serving as template for a second rolling-circle leading to the oligomeric (+) RNA intermediates that are processed into the $m c(+)$ RNA. Therefore, the second half of the cycle is symmetric with respect to the first. Most importantly, cleavage of the oligomeric RNA intermediates is autocatalytic and mediated by hammerhead ribozymes embedded in both polarity strands [50].

\subsection{Initiation and elongation: involvement of a nuclear-encoded chloroplastic RNA polymerase}

Two different RNA polymerases have been reported in plastids, the plastid-encoded polymerase (PEP) with a multisubunit structure similar to the Escherichia coli enzyme, and the single-unit nuclearencoded polymerase (NEP) resembling phage RNA polymerases. The available evidence supports the involvement of the second in replication of chloroplastic viroids. First, adding micromolar concentrations of the toxin tagetin to chloroplastic preparations from ASBVd-infected leaves prevents in vitro transcription of representative chloroplastic genes without essentially affecting synthesis of ASBVd strands [51]. And second, accumulation of a typical NEP transcript and of PLMVd strands is particularly high in areas displaying peach calico (an albinism induced by specific PLMVd variants), in which development of proplastids into chloroplasts and processing of chloroplastic rRNA precursors (and translation of plastid-encoded proteins like PEP) is impaired [52]. These latter data also suggest that other chloroplastic proteins mediating replication (see below) are also nuclearencoded.

The question of the transcription initiation sites has been undertaken by in vitro capping with [ a $\left.{ }^{32} \mathrm{P}\right]-$ GTP and guanylyl-transferase, which specifically labels the free 5'-triphosphate group characteristic of chloroplastic primary transcripts. This approach, combined with RNase protection assays, has identified the initiation sites of ASBVd $\mathrm{ml}(+)$ and $(-)$ RNAs isolated from infected avocado at similar $(\mathrm{A}+\mathrm{U})$-rich terminal loops in their predicted quasi-rod-like secondary structures [53]. The same methodology, but combined with RNA ligase-mediated rapid amplification of cDNA ends to enhance its sensitivity, has mapped the initiation sites of PLMVd $m l(+)$ and $(-)$ RNAs isolated from infected peach at similar double-stranded motifs of 6 to 7 bp that also contain the conserved GUC triplet preceding the self-cleavage site in both polarity strands; within their predicted branched secondary structures, the motifs are located at the base of similar long hairpins that presumably contain the NEP promoters [54]. These PLMVd initiation sites, which have been later confirmed [55], are different from those proposed previously on the basis of primer-extensions of the 5 ' termini of PLMVd subgenomic RNAs isolated from infected peach, and of in vitro transcriptions with truncated PLMVd RNAs and the RNA polymerase of E. coli [56]. This discrepancy most likely derives from the difficulties in reconstituting in vitro a bona fide initiation complex reproducing the in vivo situation, particularly with a eubacterial RNA polymerase very different from NEP. Therefore, the structural motifs containing the transcription initiation sites are clearly distinct in the two viroids: terminal loops in ASBVd and double-stranded motifs in PLMVd.

\subsection{Cleavage of oligomeric (+) and (-) strands is mediated by hammerhead ribozymes}

Apart from the viroid discovery itself, the finding - first in ASBVd and then in the other members of the family Avsunviroidae [12,50] — that the strands of both polarities self-cleave through 
hammerhead ribozymes has attracted much attention on these small RNA replicons, which are now regarded as remnants of the RNA world postulated to have preceded the present world on Earth based on DNA and proteins [57]. Previous descriptions of the hammerheads found in viroids and in other small RNAs, and of the evidence supporting that they mediate self-cleavage in vivo of the multimeric viroid replicative intermediates wherein they are embedded, have been reviewed elsewhere [12,50]. Here we will focus on recent advances, which once again, have provided some surprises.

Hammerhead structures are formed by a central core of conserved sequences surrounded by three double-stranded stems (I, II and III) with loose sequence requirements and usually capped by short loops (1, 2 and 3). X-ray crystallography has unveiled that the actual conformation of these catalytic motifs does not resemble a hammerhead, as suggested by the initial bidimensional representation, but rather a Y wherein stems III and II are almost colinear (Figure 4). Data obtained with model hammerheads acting in trans (an artificial design with opened loops 1 and 2 that facilitates kinetic analysis in protein-free media) showed that efficient cleavage in vitro requires $\mathrm{Mg}^{2+}$ concentrations of 5-10 $\mathrm{mM}$, an order of magnitude higher than that existing in vivo. However, re-examinations in vitro and in vivo have demonstrated that natural cis-acting hammerheads with intact loops 1 and 2 selfcleave much faster (retaining activity at $0.5-1 \mathrm{mM} \mathrm{Mg}^{2+}$ ), and that modifications of loops 1 and 2 cause a severe reduction of their catalytic activity [58,59], thus supporting that loop-loop tertiary interactions play a key role in the folding and catalytic activity of natural hammerheads. The recent Xray crystal structures of two full-length natural hammerheads indeed show that the tertiary contacts, despite being distal from the active site, promote the adoption of a catalytically active conformation by the central core $[60,61]$. Moreover, applying a combination of NMR (nuclear magnetic resonance) spectroscopy, site-directed mutagenesis and kinetic and infectivity analyses to the (+) and (-) hammerheads of CChMVd has revealed that, in both hammerheads, loop 1 is a heptanucleotide hairpin loop containing an exposed $U$ at its 5' side and an extrahelical $U$ at its 3'-side critical for the catalytic activity of the ribozyme in vitro and for viroid infectivity in vivo, whereas loop 2 has a key opened A at its 3 '-side. These structural features promote a specific loop-loop interaction motif across the major groove, the structural features of which — base pairing between the 5' pyrimidine of loop 1 and the 3' purine of loop 2, and interaction of the extrahelical pyrimidine of loop 1 with loop 2 (Figure 4) - are likely shared by a significant fraction of natural hammerheads [62].

Two additional points regarding hammerhead function in the natural context deserve a comment. First, the loop-loop tertiary interactions could be further stabilized in vivo by chloroplastic proteins acting as RNA chaperones [63]. Further dissection of the processing requirements in the family Avsunviroidae may be tackled with a system based in transplastomic Chlamydomonas reinhardtii expressing (+) and (-) dimeric transcripts; despite the absence of viroid RNA-RNA transcription, this system can be used to identify the cellular factors facilitating cleavage (and ligation, see below) [64]. And second, the differential effects in co- and post-transcriptional self-cleavage of mutations affecting the trinucleotide preceding the self-cleavage site of (+) and (-) ELVd hammerheads suggest that: i) natural hammerheads have been evolutionarily selected to function co-transcriptionally, and ii) the trinucleotide AUC preceding the self-cleavage site is most likely excluded in the majority of natural hammerheads because it promotes the transient adoption of catalytically-inactive metastable structures during transcription [65]. Therefore, hammerhead-mediated catalysis appears closely associated with RNA folding taking place during transcription. 
Figure 4. Hammerhead structures. (A) Schematic representation of a typical hammerhead structure as originally proposed [45]. Residues strictly or highly conserved in natural hammerhead structures are in yellow on a black background. Arrow marks the selfcleavage site, $\mathrm{N}$ indicates any residue and $\mathrm{H}$ any residue except $\mathrm{G}$, and continuous and broken lines denote Watson-Crick and non-canonical base pairs, respectively. (B) Hammerhead structure represented according to X-ray crystallography and NMR data [6062]. The proposed tertiary interaction between loops 1 and 2, which facilitates catalytic activity in vivo, is indicated with red broken lines. Pyr and Pur refer to pyrimidine and purine, respectively.
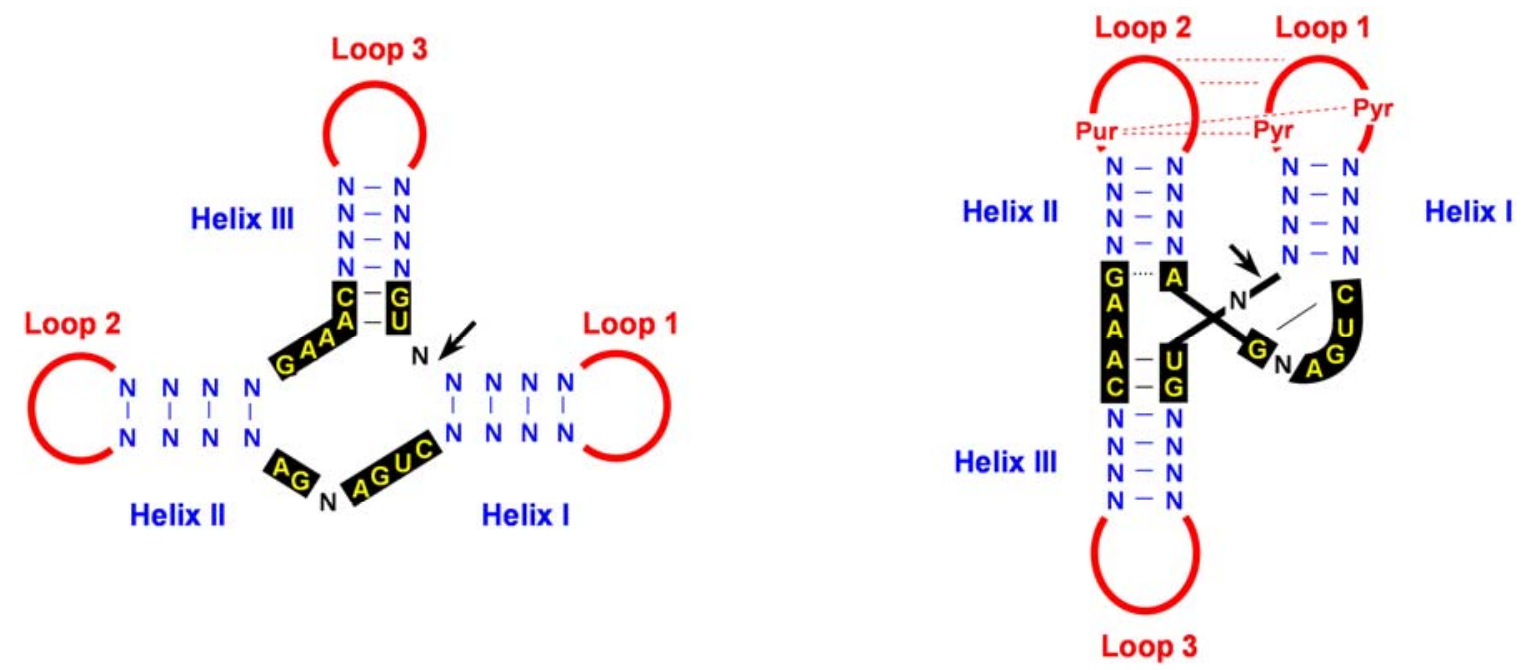

\subsection{Ligation: an unsolved question with several possible alternatives}

First, considering that the 5'-OH and 2', 3'-cyclic phosphodiester termini resulting from hammerhead-mediated self-cleavage are those typically required by the wheat germ tRNA ligase, whose homologue from A. thaliana is the only plant RNA ligase well-characterized biochemically and genetically [66], and considering also its low substrate specificity, an enzyme of this class appears an excellent candidate for catalyzing RNA ligation in chloroplastic viroids. However, pre-tRNA splicing in yeast has been assumed to occur in the nucleus and, in consonance with this view, immunofluorescence and immune electron microscopy detected the tRNA ligase in this organelle [67]. This long-held paradigm has been questioned by the finding that yeast pre-tRNA splicing takes place in the cytoplasm [68]. Most importantly, in A. thaliana and Oryza sativa two of the splicing enzymes, the tRNA ligase and 2'-phosphotransferase (which catalyzes removal the 2'-phosphomonoester group generated by the tRNA ligase), have transit signals at their N-termini and are predominantly targeted to chloroplasts and proplastids, respectively [69]. Therefore, the plant tRNA ligase now fulfills all criteria for mediating circularization of members of the family Avsunviroidae. Moreover, in one viroid-like satellite RNA the junction resulting from ligation of the two termini generated by hammerhead-mediated self-cleavage contains the expected signature of a tRNA ligase: a 2'phosphomonoester, 3', 5'-phosphodiester group [70]. Encapsidation of this viroid-like satellite RNA by the coat protein of its helper virus might have precluded removal of the 2'-phosphomonoester. In this same direction we have observed in some PLMVd and CChMVd variants a deletion of the 
nucleotide immediately after the self-cleavage site that strongly reduces in vitro self-cleavage and infectivity. We suspect that this deletion may have been introduced during reverse transcription, because it was detected only in the plus-polarity strand, and that its ultimate cause could be the presence in a fraction of the viroid population of a 2'-phosphomonoester at the nucleotide preceding the self-cleavage/ligation site, which would lead to a jumping of the reverse transcriptase [71-73]. Another possibility is the 2', 5' -phosphodiester linkage formed in the in vitro self-ligation of PLMVd, assuming that this atypical bond is indeed present in vivo (see below).

Second, the hammerhead ribozyme may mediate not only self-cleavage but also ligation generating a 3', 5'-phosphodiester linkage. This is indeed the case for another small ribozyme, the hairpin ribozyme, which is found embedded in certain viroid-like satellite RNAs [74]. When compared with the hammerhead ribozyme, the RNA ligase activity of the hairpin ribozyme is much higher. However, recent data show that the ligase activity is considerably increased in hammerheads wherein the tertiary stabilizing interaction between loops 1 and 2 is preserved [75,76]. Therefore, it is still possible that replication of chloroplastic viroids might be an RNA-mediated mechanism only requesting a host RNA polymerase.

Third, the reaction could also be autocatalytic (self-ligation) but operating through a different pathway. This hypothesis is based on the observation that the monomeric linear PLMVd RNAs resulting from hammerhead-mediated self-cleavage can self-ligate in vitro mostly producing 2', 5'instead of the conventional 3', 5'-phosphodiester bonds [77], and on the proposal that these atypical bonds exist in circular PLMVd RNAs isolated from infected tissue and impede their in vitro selfcleavage [78]. However, this may not well be the physiological mechanism because: (i) in vitro selfligation of PLMVd demands concentrations of $\mathrm{Mg}^{+2}$ around $100 \mathrm{mM}$, which are much higher than those existing in vivo, ii) in vitro self-ligation has also been observed in PSTVd [23] and in HDV RNA, in this latter case also generating 2', 5'-phosphodiester bonds [79], while neither PSTVd nor HDV RNA appear to follow this route in vivo [26,32,80], and iii) there are no previous reports on the existence of natural RNAs with 2',5'-phosphodiester bonds serving as transcription templates; indeed, this atypical linkage blocks elongation catalyzed by the reverse transcriptase in vitro [77] and presumably by the NEP in vivo, while we have not observed prominent stops at the ligation site in our primer extension experiments using ASBVd circular forms isolated from infected tissue [81].

\section{Concluding remarks and perspectives}

The lack of protein-coding capacity of viroids entails that their replication mechanism is much more host-reliant than that of RNA viruses, which at least encode a subunit of the RNA-dependent RNA polymerase catalyzing initiation and elongation of viral strands. Moreover, viroids are able to redirect certain nuclear and chloroplastic RNA polymerases to transcribe RNA instead of their physiological DNA templates. Although the nature of the RNA polymerases involved in viroid replication appears well-established, they most likely act in concert with other host proteins that facilitate their template switching and processivity. New research avenues include identification of these proteins, as well as of the RNase mediating cleavage of oligomeric $(+)$ replicative intermediates in the family Pospiviroidae and of the RNA ligases catalyzing circularization in both families (if this step is indeed an enzymemediated reaction in the family Avsunviroidae). 
On the other hand, viroid replication may be closely linked to other biological features. Here are three illustrative examples. First, is viroid pathogenesis dependent or independent of replication? Although there is a report indicating that the ectopic expression of non-replicating viroid sequences incites typical symptoms [82], a recent work has failed to reproduce these results [83]. Therefore, symptoms may well be an effect of viroid replication and, more specifically, of detracting from their normal roles for some enzyme(s) of the RNA silencing pathways. Second, for the host range of members of the family Avsunviroidae - essentially limited to the natural hosts in which they were first reported [12,49], is it a consequence of restrictions in the access of these RNAs to the chloroplast or in their replication once they have been translocated into this organelle? And third, for the extremely high mutation rate of CChMVd, the highest reported for any biological entity [84], is it a result of being replicated by a proofreading-deficient chloroplastic DNA-dependent RNA polymerase redirected to using RNA? In other words, do other chloroplastic viroids have similar mutation rates as their rapid accumulation of sequence heterogeneity suggests? And what happens with nuclear viroids that are replicated by a different RNA polymerase and display a considerably lower sequence heterogeneity?

\section{Acknowledgements}

We are grateful to A. Ahuir for excellent technical assistance. This work was supported by the Ministerio de Ciencia e Innovación of Spain (grants BFU2008-03154/BMC to R.F. and BIO200801986 to J.-A.D.) and by the Generalidad Valenciana (ACOMP/2009/151 to R.F.).

\section{References}

1. Gross, H.J.; Domdey, H.; Lossow, C.; Jank, P.; Raba, M.; Alberty, H.; Sänger, H.L. Nucleotide sequence and secondary structure of potato spindle tuber viroid. Nature 1978, 273, 203-208.

2. Diener, T.O. Discovering viroids - a personal perspective. Nat. Rev. Microbiol. 2003, 1, 75-80.

3. Tabler, M.; Tsagris, M. Viroids: petite RNA pathogens with distinguished talents. Trends Plant Sci. 2004, 9, 339-348.

4. Flores, R.; Hernández, C.; Martínez de Alba, E.; Daròs, J.A.; Di Serio, F. Viroids and viroid-host interactions. Annu. Rev. Phytopathol. 2005, 43, 117-139.

5. Daròs, J.A.; Elena, S.F.; Flores, R. Viroids: an Ariadne's thread into the RNA labyrinth. EMBO Rep. 2006, 7, 593-598.

6. Ding, B.; Itaya, A. Viroid: a useful model for studying the basic principles of infection and RNA biology. Mol. Plant-Microb. Interact. 2007, 20, 7-20.

7. Ding, B. The biology of viroid-host interactions. Annu. Rev. Phytopathol. 2009, 47, 105-131.

8. Astier-Manifacier, S.; Cornuet, P. RNA-dependent RNA polymerase in Chinese cabbage. Biochim. Biophys. Acta 1971, 232, 484-493.

9. Schiebel, W.; Haas, B.; Marinkovic, S.; Klanner, A.; Sanger, H. L. RNA-directed RNA polymerase from tomato leaves. 1. Purification and physical-properties. J. Biol. Chem. 1993, 268, 11851-11857.

10. Przybilski, R.; Graf, S.; Lescoute, A.; Nellen, W.; Westhof, E.; Steger, G.; Hammann, C. Functional hammerhead ribozymes naturally encoded in the genome of Arabidopsis thaliana. Plant Cell 17, 1877-1885. 
11. Martick, M.; Horan, L.H.; Noller, H.F.; Scott, W.G. A discontinuous hammerhead ribozyme embedded in a mammalian messenger RNA. Nature 2008, 454, 899-902.

12. Flores, R.; Daròs, J.A.; Hernández, C. The Avsunviroidae family: viroids with hammerhead ribozymes. Adv. Virus Res. 2000, 55, 271-323.

13. Symons, R.H.; Randles, J.W. Encapsidated circular viroid-like satellite RNAs (virusoids) of plants. Curr. Top. Microbiol. Immunol. 1999, 239, 81-105.

14. Lai, M.M.C. RNA replication without RNA-dependent RNA polymerase: surprises from hepatitis delta virus. J. Virol. 2005, 79, 7951-7958.

15. Branch, A.D.; Robertson, H.D. A replication cycle for viroids and other small infectious RNAs. Science 1984, 223, 450-454.

16. Branch A.D.; Benenfeld, B.J.; Robertson, H.D. Evidence for a single rolling circle in the replication of potato spindle tuber viroid. Proc. Natl. Acad. Sci. USA 1988, 85, 9128-9132.

17. Zhong, X.; Archual, A.J.; Amin, A.A.; Ding, B.A genomic map of viroid RNA motifs critical for replication and systemic trafficking. Plant Cell 2008, 20, 35-47.

18. Kolonko, N.; Bannach, O.; Aschermann, K.; Hu, K.H.; Moors, M.; Schmitz, M.; Steger, G.; Riesner, D. Transcription of potato spindle tuber viroid by RNA polymerase II starts in the left terminal loop. Virology 2006, 347, 392-404.

19. Riesner, D.; Henco, K.; Rokohl, U.; Klotz, G.; Kleinschmidt, A.K.; Domdey, H.; Jank, P.; Gross, H.J.; Sänger, H.L. Structure and structure formation of viroids. J. Mol. Biol. 1979, 133, 85-115.

20. Tabler, M.; Sänger, H.L. Infectivity studies on different potato spindle tuber viroid (PSTV) RNAs synthesized in vitro with the SP6 transcription system. EMBO J. 1985, 4, 2191-2199.

21. Meshi, T.; Ishikawa, M; Watanabe, Y.; Yamaya, J.; Okada, Y., Sano, T.; Shikata, E. The sequence necessary for the infectivity of hop stunt viroid cDNA clones. Mol. Gen. Genet. 1985, 200, 199206.

22. Visvader, J.E.; Forster, A.C.; Symons, R.H. Infectivity and in vitro mutagenesis of monomeric cDNA clones of citrus exocortis viroid indicates the site of processing of viroid precursors. Nucleic Acids Res. 1985, 13, 5843-5856.

23. Diener, T.O. Viroid processing: a model involving the central conserved region and hairpin I. Proc. Natl. Acad. Sci. USA 1986, 83, 58-62.

24. Baumstark, T.; Schröder, A.R.; Riesner, D. Viroid processing: switch from cleavage to ligation is driven by a change from a tetraloop to a loop E conformation. EMBO J. 1997, 16, 599-610.

25. Flores, R.; Di Serio, F.; Hernández, C. Viroids: the noncoding genomes. Semin. Virol. 1997, 8, 6573.

26. Gas, M.E.; Hernández, C.; Flores, R.; Daròs, J.A. Processing of nuclear viroids in vivo: an interplay between RNA conformations. PLoS Pathog. 2007, 3, 1813-1826.

27. Daròs, J.A.; Flores, R. Arabidopsis thaliana has the enzymatic machinery for replicating representative viroid species of the family Pospiviroidae. Proc. Natl. Acad. Sci. USA 2004, 101, 6792-6797.

28. Gast, F.U.; Kempe, D.; Sänger, H.L. The dimerization domain of potato spindle tuber viroid, a possible hallmark for infectious RNA. Biochemistry 1998, 37, 14098-14107.

29. Bernstein, E.; Caudy, A.A.; Hammond, S.M.; Hannon, G.J. Role for a bidentate ribonuclease in the initiation step of RNA interference. Nature 2001, 409, 363-366. 
30. MacRae, I.J.; Doudna, J.A. Ribonuclease revisited: structural insights into ribonuclease III family enzymes. Curr. Opin. Struct. Biol. 2007, 17, 1-8.

31. Hiraguri, A.; Itoh, R.; Kondo, N.; Nomura, Y.; Aizawa, D.; Murai, Y.; Koiwa, H.; Seki, M.; Shinozaki, K.; Fukuhara, T. Specific interactions between Dicer-like proteins and HYL1/DRBfamily dsRNA-binding proteins in Arabidopsis thaliana. Plant Mol. Biol. 2005, 57, 173-188.

32. Gas, M.E.; Molina-Serrano, D.; Hernández, C.; Flores, R.; Daròs, J.A. Monomeric linear RNA of citrus exocortis viroid resulting from processing in vivo has 5'-phosphomonoester and 3'-hydroxyl termini: implications for the ribonuclease and RNA ligase involved in replication. J. Virol. 2008, 82, 10321-10325.

33. Paillart, J.C.; Shehu-Xhilaga, M.; Marquet, R.; Mak, J. Dimerization of retroviral RNA genomes: an inseparable pair. Nat. Rev. Microbiol. 2004, 2, 461-472.

34. Branch, A.D.; Robertson, H.D.; Greer, C.; Gegenheimer, P.; Peebles, C.; Abelson, J. Cell-free circularization of viroid progeny RNA by an RNA ligase from wheat germ. Science 1982, 217, 1147-1149.

35. Konarska, M.; Filipowicz, W.; Domdey, H.; Gross, H.J. Formation of a 2'-phosphomonoester, 3',5'-phosphodiester linkage by a novel RNA ligase in wheat germ. Nature 1981, 293, 112-116.

36. Tabler, M.; Tzortzakaki, S.; Tsagris, M. Processing of linear longer-than-unit-length potato spindle tuber viroid RNAs into infectious monomeric circular molecules by a G-specific endoribonuclease. Virology 1992, 190, 746-753.

37. Branch, A.D.; Benenfeld, B.J.; Robertson, H.D. Ultraviolet light-induced crosslinking reveals a unique region of local tertiary structure in potato spindle tuber viroid and HeLa 5S RNA. Proc. Natl. Acad. Sci. USA 1985, 82, 6590-6594.

38. Eiras, M.; Kitajima, E.W.; Flores, R.; Daròs, J.A. Existence in vivo of the loop E motif in potato spindle tuber viroid RNA. Arch. Virol. 2007, 152, 1389-1393.

39. Wang, Y.; Zhong, X.; Itaya, A.; Ding, B. Evidence for the existence of the loop E motif of potato spindle tuber viroid in vivo. J. Virol. 2007, 81, 2074-2077.

40. Wassenegger, M.; Spieker, R.L.; Thalmeir, S.; Gast, F.U.; Riedel, L.; Sänger, H.L. A single nucleotide substitution converts potato spindle tuber viroid (PSTVd) from a noninfectious to an infectious RNA for Nicotiana tabacum. Virology 1996, 226, 191-197.

41. Qi, Y.; Ding, B. Inhibition of cell growth and shoot development by a specific nucleotide sequence in a noncoding viroid RNA. Plant Cell 2003, 15, 1360-1374.

42. Zhong, X.; Leontis, N.; Qiang, S.; Itaya, A.; Qi, Y.; Boris-Lawrie, K.; Ding, B. Tertiary structural and functional analysis of a viroid RNA motif by isostericity matrix and mutagenesis reveal its essential role in replication. J. Virol. 2006, 80, 8566-8581.

43. Ho, C.K.; Shuman, S. Bacteriophage T4 RNA ligase 2 (gp24.1) exemplifies a family of RNA ligases found in all phylogenetic domains. Proc. Natl. Acad. Sci. USA 2002, 99, 12709-12714.

44. Wang, L.K.; Schwer, B.; Shuman, S. Structure-guided mutational analysis of T4 RNA ligase 1. RNA 2006, 12, 2126-2134.

45. Hutchins, C.; Rathjen, P.D.; Forster, A.C; Symons, R.H. Self-cleavage of plus and minus RNA transcripts of avocado sunblotch viroid. Nucleic Acids Res. 1986, 14, 3627-3640. 
46. Daròs, J.A.; Marcos, J.F.; Hernández, C.; Flores, R. Replication of avocado sunblotch viroid: evidence for a symmetric pathway with two rolling circles and hammerhead ribozyme processing. Proc. Natl. Acad. Sci. USA 1994, 91, 12813-12817.

47. Hernández, C.; Flores, R. Plus and minus RNAs of peach latent mosaic viroid self-cleave in vitro via hammerhead structures. Proc. Natl. Acad. Sci. USA 1992, 89, 3711-3715.

48. Navarro, B.; Flores, R. Chrysanthemum chlorotic mottle viroid: unusual structural properties of a subgroup of viroids with hammerhead ribozymes. Proc. Natl. Acad. Sci. USA 1997, 94, $11262-$ 11267.

49. Fadda, Z.; Daròs, J.A.; Fagoaga, C.; Flores, R.; Duran-Vila, N. Eggplant latent viroid (ELVd): candidate type species for a new genus within family Avsunviroidae (hammerhead viroids). J. Virol. 2003, 77, 6528-6532.

50. Flores, R.; Hernández, C.; De la Peña, M.; Vera, A.; Daròs, J.A. Hammerhead ribozyme structure and function in plant RNA replication. Methods Enzymol. 2001, 341, 540-552.

51. Navarro, J.A.; Vera, A.; Flores, R.A chloroplastic RNA polymerase resistant to tagetitoxin is involved in replication of avocado sunblotch viroid. Virology 2000, 268, 218-225.

52. Rodio, M.E.; Delgado, S.; De Stradis, A.E.; Gómez, M.D.; Flores, R.; Di Serio, F. A viroid RNA with a specific structural motif inhibits chloroplast development. Plant Cell 2007, 19, 3610-3626.

53. Navarro, J.A.; Flores, R. Characterization of the initiation sites of both polarity strands of a viroid RNA reveals a motif conserved in sequence and structure. EMBO J. 2000, 19, 2662-2670.

54. Delgado, S.; Martínez de Alba, E.; Hernández, C.; Flores, R. A short double-stranded RNA motif of peach latent mosaic viroid contains the initiation and the self-cleavage sites of both polarity strands. J. Virol. 2005, 79, 12934-12943.

55. Motard, J.; Bolduc, F.; Thompson, D.; Perreault, J.P. The peach latent mosaic viroid replication initiation site is located at a universal position that appears to be defined by a conserved sequence. Virology 2008, 373, 362-375.

56. Pelchat, M.; Côté, F.; Perreault, J.P. Study of the polymerization step of the rolling circle replication of peach latent mosaic viroid. Arch. Virol. 2001, 146, 1753-1763.

57. Diener, T.O. Circular RNAs: relics of precellular evolution? Proc. Natl. Acad. Sci. USA 1989, 86, 9370-9374.

58. De la Peña, M.; Gago, S.; Flores, R. Peripheral regions of natural hammerhead ribozymes greatly increase their self-cleavage activity. EMBO J. 2003, 22, 5561-5570.

59. Khvorova, A.; Lescoute, A.; Westhof, E.; Jayasena, S.D. Sequence elements outside the hammerhead ribozyme catalytic core enable intracellular activity. Nat. Struct. Biol. 2003, 10, 708712 .

60. Martick, M.; Scott, W.G. Tertiary contacts distant from the active site prime a ribozyme for catalysis. Cell 2006, 126, 309-320.

61. Chi, Y.I.; Martick, M.; Lares, M.; Kim, R.; Scott, W.G.; Kim, S.H. Capturing hammerhead ribozyme structures in action by modulating general base catalysis. PLoS Biol. 2008, 6, 20602068.

62. Dufour, D.; De la Peña, M.; Gago, S.; Flores, R.; Gallego, J. Structure-function analysis of the ribozymes of chrysanthemum chlorotic mottle viroid: a loop-loop interaction motif conserved in most natural hammerheads. Nucleic Acids Res. 2009, 37, 368-381. 
63. Daròs, J.A.; Flores, R.A chloroplast protein binds a viroid RNA in vivo and facilitates its hammerhead-mediated self-cleavage. EMBO J. 2002, 21, 749-759.

64. Molina-Serrano, D.; Suay, L.; Salvador, M.L.; Flores, R.; Daròs, J.A. Processing of RNAs of the family Avsunviroidae in Chlamydomonas reinhardtii chloroplasts. J. Virol. 2007, 81, 4363-4366.

65. Carbonell, A.; De la Peña, M.; Flores, R.; Gago, S. Effects of the trinucleotide preceding the selfcleavage site on eggplant latent viroid hammerheads: differences in co- and post-transcriptional self-cleavage may explain the lack of trinucleotide AUC in most natural hammerheads. Nucleic Acids Res. 2006, 34, 5613-5622.

66. Englert, M; Beier, H. Plant tRNA ligases are multifunctional enzymes that have diverged in sequence and substrate specificity from RNA ligases of other phylogenetic origins. Nucleic Acids Res. 2005, 33, 388-399.

67. Clark, M.W.; Abelson, J. The subnuclear localization of transfer-RNA ligase in yeast. J. Cell Biol. 1987, 105, 1515-1526.

68. Yoshihisa, T; Yunoki-Esaki, K; Ohshima, C.; Tanaka, N.; Endo, T. Possibility of cytoplasmic pretRNA splicing: the yeast tRNA splicing endonuclease mainly localizes on the mitochondria. Mol. Biol. Cell 2003, 14, 3266-3279.

69. Englert, M; Latz, A; Becker, D.; Gimple, O.; Beier, H.; Akama, K. Plant pre-tRNA splicing enzymes are targeted to multiple cellular compartments. Biochimie 2007, 89, 1351-1365.

70. Kibertis, P.A.; Haseloff, J.; Zimmern, D. 2' phosphomonoester, 3'-5' phosphodiester bond at a unique site in a circular viral RNA. EMBO J. 1985, 4, 817-827.

71. Ambrós, S.; Hernández, C.; Desvignes, J.C; Flores, R. Genomic structure of three phenotypically different isolates of peach latent mosaic viroid: Implications of the existence of constraints limiting the heterogeneity of viroid quasi-species. J. Virol. 1998, 72, 7397-7406.

72. Ambrós, S., Hernández, C. Flores, R. Rapid generation of genetic heterogeneity in progenies from individual cDNA clones of peach latent mosaic viroid in its natural host. J. Gen. Virol. 1999, 80, 2239-2252.

73. De la Peña, M., Navarro, B., Flores, R. Mapping the molecular determinant of pathogenicity in a hammerhead viroid: a tetraloop within the in vivo branched RNA conformation. Proc. Natl. Acad. Sci. USA 1999, 96, 9960-9965.

74. Buzayan, J.M.; Gerlach, W.L.; Bruening, G. Nonenzymatic cleavage and ligation of RNAs complementary to a plant-virus satellite RNA. Nature 1986, 323, 349-353.

75. Stage-Zimmermann, T.K.; Uhlenbeck, O.C. A covalent crosslink converts the hammerhead ribozyme from a ribonuclease to an RNA ligase. Nat. Struct. Biol. 2001, 8, 863-867.

76. Nelson, J.A.; Shepotinovskaya, I.; Uhlenbeck, O.C. Hammerheads derived from sTRSV show enhanced cleavage and ligation rate constants. Biochemistry 2005, 44, 14577-14585.

77. Côté, F.; Perreault, J.P. Peach latent mosaic viroid is locked by a 2',5'- phosphodiester bond produced by in vitro self-ligation. J. Mol. Biol. 1997, 273, 533-543.

78. Côté, F.; Lévesque, D.; Perreault, J.P. Natural 2',5'-phosphodiester bonds found at the ligation sites of peach latent mosaic viroid. J. Virol. 2001, 75, 19-25.

79. Sharmeen, L.; Kuo, M.Y.P.; Taylor, J. Self-ligating RNA sequences on the antigenome of human hepatitis delta-virus. J. Virol. 1989, 63, 1428-1430. 
80. Reid, C.E; Lazinski, D.W. A host-specific function is required for ligation of a wide variety of ribozyme-processed RNAs. Proc. Natl. Acad. Sci. USA 2000, 97, 424-429.

81. Marcos, J.F.; Flores, R. The 5' end generated in the in vitro self-cleavage reaction of avocado sunblotch viroid RNAs is present in naturally occurring linear viroid molecules. J. Gen. Virol. 1993, 74, 907-910.

82. Wang, M.B.; Bian, X.Y.; Wu, L.M.; Liu, L.X.; Smith, N.A.; Isenegger, D.; Wu, R.M.; Masuta, C.; Vance, V.B.; Watson, J.M.; Rezaian, A.; Dennis, E.S.; Waterhouse, P.M. On the role of RNA silencing in the pathogenicity and evolution of viroids and viral satellites. Proc. Natl. Acad. Sci. USA 2004, 101, 3275-3280.

83. Schwind, N.; Zwiebel, M.; Itaya, A.; Ding, B.; Wang, M.B.; Krczal, G.; Wassenegger, M. RNAimediated resistance to potato spindle tuber viroid in transgenic tomato expressing a viroid hairpin RNA construct. Mol. Plant Pathol. 2009, 10, 459-469.

84. Gago, S.; Elena, S.F.; Flores, R.; Sanjuán, R. Extremely high variability of a hammerhead viroid. Science 2009, 323, 1308.

(C) 2009 by the authors; licensee Molecular Diversity Preservation International, Basel, Switzerland. This article is an open-access article distributed under the terms and conditions of the Creative Commons Attribution license (http://creativecommons.org/licenses/by/3.0/). 